\title{
LINEAR TRANSFORMATIONS PRESERVING POTENT MATRICES
}

\author{
MATEJ BREŠAR AND PETER ŠEMRL
}

(Communicated by Maurice Auslander)

\begin{abstract}
Linear transformations of $M_{n}$, the algebra of $n \times n$ matrices over $\mathbb{C}$, which preserve the set of all potent matrices, are characterized.
\end{abstract}

Let $M_{n}$ be the algebra of $n \times n$ matrices over a field. A number of authors have characterized linear transformations $\theta$ on $M_{n}$ which preserve some subsets $\Gamma$ of $M_{n}$ (i.e., $\theta(\Gamma) \subseteq \Gamma$ ). Let us list some examples of such subsets: the case when $\Gamma$ is the set of all singular matrices was considered by Dieudonné [3]; $\Gamma=\left\{A \in M_{n} \mid \operatorname{rank} A \leq 1\right\}$ by Jacob [7] and Marcus and Moyls [9]; $\Gamma$ is a linear group by Dixon [4]; and $\Gamma$ is the set of all nilpotent matrices by Botta, Pierce, and Watkins [1]. In a recent paper [2], motivated by a problem of characterizing local automorphisms and local derivations of some operator algebras (see, e.g., [8]), the present authors considered the case when $\Gamma$ is the set of all projections in $M_{n}$. In this paper, we consider a more general situation; namely, we deal with linear transformations preserving potent matrices (recall that a matrix $A$ is said to be potent if $A^{r}=A$ for some integer $r \geq 2$ ). The set of all potent matrices will be denoted by $\pi$. For any integer $r \geq 2$ we define $\pi_{r}=\left\{A \in M_{n} \mid A^{r}=A\right\}$. By $A^{\mathrm{t}}$ and $\operatorname{tr}(A)$ we denote the transpose and the trace of $A$, respectively. The aim of this paper is to prove the following

Theorem. Let $M_{n}$ be the algebra of $n \times n$ matrices over the complex field $\mathbb{C}$, and let $\theta \neq 0$ be a linear transformation on $M_{n}$. The following conditions are equivalent.

(i) $\theta(\pi) \subseteq \pi$.

(ii) There exists an integer $r \geq 2$ such that $\theta\left(\pi_{r}\right) \subseteq \pi_{r}$.

(iii) $\theta$ is either of the form

$$
\theta(A)=c U A U^{-1} \text { or } \theta(A)=c U A^{t} U^{-1},
$$

where $U \in M_{n}$ is an invertible matrix and $c \in \mathbb{C}$ is a root of unity.

Proof. It is clear that (iii) implies (i) and (ii). We shall prove the converse implications.

First, assume that $\theta\left(\pi_{r}\right) \subseteq \pi_{r}$ for some $r \geq 2$, and let us show that (iii) holds. Let $P, Q \in M_{n}$ be orthogonal projections (i.e., $P^{2}=P, Q^{2}=Q$,

Received by the editors February 3, 1992.

1991 Mathematics Subject Classification. Primary 15A04.

Key words and phrases. Linear transformation, potent matrix. 
and $P Q=Q P=0)$. Let $\lambda_{1}, \lambda_{2}, \ldots, \lambda_{r-1}$ be $(r-1)$-roots of unity, and note that $P+\lambda_{i} Q \in \pi_{r}, i=1,2, \ldots, r-1$. By the assumption it follows that $\theta\left(P+\lambda_{i} Q\right)^{r}=\theta\left(P+\lambda_{i} Q\right) ;$ that is,

$$
\left(A+\lambda_{i} B\right)^{r}=A+\lambda_{i} B,
$$

where $A=\theta(P)$ and $B=\theta(Q)$. As $A^{r}=A$ and $B^{r}=B$ (namely, $P$ and $Q$ belong to $\pi_{r}$ ), this relation can be written in the form

$$
\lambda_{i} C_{1}+\lambda_{i}^{2} C_{2}+\cdots+\lambda_{i}^{r-1} C_{r-1}=0, \quad i=1,2, \ldots, r-1,
$$

where

$$
\begin{aligned}
C_{1} & =A^{r-1} B+A^{r-2} B A+\cdots+A B A^{r-2}+B A^{r-1}, \\
C_{2} & =A^{r-2} B^{2}+A^{r-3} B A B+\cdots+B^{2} A^{r-2}, \\
& \vdots \\
C_{r-1} & =A B^{r-1}+B A B^{r-2}+\cdots+B^{r-2} A B+B^{r-1} A .
\end{aligned}
$$

Since the $\lambda_{i}$ 's are nonzero and mutually different, it follows that $C_{1}=C_{2}=$ $\cdots=C_{r-1}=0$; thus, in particular,

$$
A^{r-1} B+A^{r-2} B A+\cdots+A B A^{r-2}+B A^{r-1}=0 .
$$

Multiply this relation first from the left by $A$ and then from the right by $A$. Comparing the two relations so obtained and using $A^{r}=A$, we get that $A$ and $B$ commute. Hence $A^{r-1} B=0$ and, therefore, $A B=A^{r} B=0$.

Thus, we proved the following: If $P$ and $Q$ are orthogonal projections then $\theta(P) \theta(Q)=\theta(Q) \theta(P)=0$. Now pick a selfadjoint matrix $S$. There exist mutually orthogonal projections $P_{1}, P_{2}, \ldots, P_{n}$ and real numbers $t_{1}, t_{2}, \ldots, t_{n}$ such that $S=\sum_{. i=1}^{n} t_{i} P_{i}$. Since $\theta\left(P_{i}\right) \theta\left(P_{j}\right)=\theta\left(P_{j}\right) \theta\left(P_{i}\right)=0, i \neq j$, and $\theta\left(P_{i}\right)^{r}=\theta\left(P_{i}\right)$, it follows that

$$
\theta\left(S^{r}\right)=\sum_{i=1}^{n} t_{i}^{r} \theta\left(P_{i}\right)=\theta(S)^{r} .
$$

Set $K=\theta(I)$. In the relation $\theta\left(S^{r}\right)=\theta(S)^{r}$, replace $S$ by $S+t I$, where $t$ is a real number. Then we get

$$
\begin{aligned}
\theta\left(S^{r}\right)+ & r t \theta\left(S^{r-1}\right)+\cdots+(r(r-1) / 2) t^{r-2} \theta\left(S^{2}\right)+r t^{r-1} \theta(S)+t^{r} K \\
= & \theta\left((S+t I)^{r}\right)=(\theta(S)+t K)^{r} \\
= & \theta(S)^{r}+t\left(\theta(S)^{r-1} K+\theta(S)^{r-2} K \theta(S)+\cdots+K \theta(S)^{r-1}\right) \\
& +\cdots+t^{r-2}\left(\theta(S)^{2} K^{r-2}+\theta(S) K \theta(S) K^{r-3}+\cdots+K^{r-2} \theta(S)^{2}\right) \\
& +t^{r-1}\left(\theta(S) K^{r-1}+K \theta(S) K^{r-2}+\cdots+K^{r-1} \theta(S)\right)+t^{r} K^{r} .
\end{aligned}
$$

Comparing the coefficients at $t^{r-1}$, we obtain

$$
r \theta(S)=\theta(S) K^{r-1}+K \theta(S) K^{r-2}+\cdots+K^{r-1} \theta(S) .
$$

Multiplying this relation first from the left by $K$ and then from the right by $K$ and using $K^{r}=K$, it follows that $\theta(S) K=K \theta(S)$. Consequently, comparing the coefficients at $t^{r-2}$, we arrive at

$$
\theta\left(S^{2}\right)=K^{r-2} \theta(S)^{2},
$$


where $S$ is an arbitrary selfadjoint matrix. Define a transformation $\varphi$ on $M_{n}$ by

$$
\varphi(A)=K^{r-2} \theta(A) .
$$

For a selfadjoint $S \in M_{n}$ we then have

$$
\varphi\left(S^{2}\right)=K^{r-2} \theta\left(S^{2}\right)=K^{2 r-4} \theta(S)^{2},
$$

and, since $\theta(S)$ commutes with $K$,

$$
\varphi(S)^{2}=\left(K^{r-2} \theta(S)\right)^{2}=K^{2 r-4} \theta(S)^{2} .
$$

Thus $\varphi\left(S^{2}\right)=\varphi(S)^{2}$. Replacing $S$ by $S+T$, where $S$ and $T$ are selfadjoint, we then get

$$
\varphi(S T+T S)=\varphi(S) \varphi(T)+\varphi(T) \varphi(S) .
$$

Since every $X \in M_{n}$ can be written in the form $X=S_{1}+i S_{2}$ with $S_{1}, S_{2}$ selfadjoint, it follows that

$$
\varphi(X Y+Y X)=\varphi(X) \varphi(Y)+\varphi(Y) \varphi(X)
$$

holds for all $X, Y \in M_{n}$; thus, $\varphi$ is a Jordan homomorphism. We claim that either $\varphi$ is nonsingular or $\varphi=0$. Indeed, $\operatorname{Ker} \varphi$ is a Jordan ideal (i.e., $\operatorname{Ker} \varphi$ is a linear subspace such that $A \in \operatorname{Ker} \varphi$ implies $A X+X A \in \operatorname{Ker} \varphi$ for every $X \in M_{n}$ ), but then $\operatorname{Ker} \varphi=M_{n}$ or $\operatorname{Ker} \varphi=\{0\}$ (cf. [6, Theorem 1.1]). Suppose $\varphi=0$. Then we have $0=\varphi(I)=K^{r-2} \theta(I)=K^{r-1}$ and, therefore, $K=K^{r}=0$. We showed that for any projection $P$ we have $\theta(P) \theta(I-P)=0$, so $K=0$ gives $\theta(P)^{2}=0$, and thus $\theta(P)=\theta(P)^{r}=0$. Since every matrix can be written as a linear combination of projections, it follows that $\theta=0$, contrary to the assumption. Thus $\varphi \neq 0$, so $\varphi$ is nonsingular; but then $\varphi$ is either an automorphism or an antiautomorphism [6, Theorem 3.1]. It is well known that in the first case it is of the form $\varphi(A)=U A U^{-1}$ for some invertible $U$ in $M_{n}$, and in the second case, $\varphi(A)=U A^{t} U^{-1}$.

Recall that $\varphi(A)=K^{r-2} \theta(A)$. As $\varphi$ is nonsingular, $\theta$ must be nonsingular, too. We proved that $K$ commutes with $\theta(S)$ for every selfadjoint matrix $S$; this clearly yields that $K$ commutes with $\theta(A)$ for any $A \in M_{n}$. Since $\theta$ is nonsingular, it follows that $K=c I$ for some $c \in \mathbb{C}$. Using $K^{r}=K$ and $K \neq 0$ we get $c^{r-1}=1$; hence, $\theta(A)=c \varphi(A)$. The proof of the assertion that (ii) implies (iii) is thereby completed.

Now we come to the central part of the proof; namely, we shall prove that (i) implies (iii). Let us first point out two simple observations which will be used repeatedly. A matrix $A$ is potent if and only if $A$ is diagonable and its eigenvalues are either roots of unity or 0 . Therefore, every upper triangular matrix with mutually different roots of unity on the diagonal is potent.

We divide the proof into eight steps.

Step 1. If $N \in M_{n}$ is nilpotent then $\theta(N)$ is nilpotent.

Proof of Step 1. There exists an invertible $S \in M_{n}$ such that $S N S^{-1}$ is strictly upper triangular. Let $R$ be a diagonal matrix with mutually different roots of unity on a diagonal. Then $R+\alpha S N S^{-1}$ is a potent matrix for any $\alpha \in \mathbb{C}$. Therefore, the same is true for matrices $R_{1}+\alpha N, \alpha \in \mathbb{C}$, where $R_{1}=S^{-1} R S$; that is, for any $\alpha \in \mathbb{C}$ there exists an integer $n_{\alpha} \geq 2$ such that

$$
\left(\theta\left(R_{1}\right)+\alpha \theta(N)\right)^{n_{c}}=\theta\left(R_{1}\right)+\alpha \theta(N) .
$$


Clearly, there exists an integer $n_{0} \geq 2$ such that

$$
\left(\theta\left(R_{1}\right)+\alpha \theta(N)\right)^{n_{0}}=\theta\left(R_{1}\right)+\alpha \theta(N)
$$

holds for infinitely many $\alpha$, but then this must be fulfilled for every $\alpha \in \mathbb{C}$; thus, we have

$$
\begin{aligned}
\theta(N)^{n_{0}} & =\lim _{|\alpha| \rightarrow \infty}\left(\alpha^{-1} \theta\left(R_{1}\right)+\theta(N)\right)^{n_{0}} \\
& =\lim _{|\alpha| \rightarrow \infty}\left(\alpha^{-n_{0}} \theta\left(R_{1}\right)+\alpha^{-n_{0}+1} \theta(N)\right)=0 .
\end{aligned}
$$

Step 2. There exists $c \in \mathbb{C}$ such that $\operatorname{tr}(\theta(A))=c \operatorname{tr}(A)$.

Proof of Step 2. It is easy to see that the linear span of all nilpotent matrices is the space $\mathrm{sl}_{n}$ of matrices with trace zero (namely, denoting by $E_{i j}$ the matrix whose only nonzero entry is 1 in a position $(i, j)$, we see that the nilpotent matrices $E_{i j}$ and $E_{i i}+E_{i j}-E_{j i}-E_{j j}$ for $i \neq j$ span $\left.\operatorname{sl}_{n}\right)$. In view of Step 1, we then have $\theta\left(\mathrm{sl}_{n}\right) \subseteq \mathrm{sl}_{n}$. Since for any $A \in M_{n}$ the matrix $A-(\operatorname{tr}(A) / n) I$ belongs to $\mathrm{sl}_{n}$, it follows that $\operatorname{tr}(\theta(A-(\operatorname{tr}(A) / n) I))=0$; that is, $\operatorname{tr}(\theta(A))=c \operatorname{tr}(A)$, where $c=\operatorname{tr}(\theta(I)) / n$.

Step 3. $\theta(I) \neq 0$.

Proof of Step 3. Suppose $\theta(I)=0$. Let $P$ be a projection. Since $I-P$ and $I-2 P$ are both potents, it follows that $-\theta(P)=\theta(I-P)$ and $-2 \theta(P)=$ $\theta(I-2 P)$ are potents too; but, then $\theta(P)=0$. Since the linear span of all projections is $M_{n}$, it follows, contrary to the assumption, that $\theta=0$.

Step 4. If $A \in \pi$ and $A \neq 0$, then $\theta(A) \neq 0$.

Proof of Step 4. Since $A \in \pi$, we have $A=R\left(\sum_{i=1}^{k} \lambda_{i} E_{i i}\right) R^{-1}$, where $1 \leq k \leq$ $n, \lambda_{i}$ 's are roots of unity, and $R$ is an invertible matrix. Suppose that $\theta(A)=$ 0 . Obviously, for any $i \in\{1,2, \ldots, k\}$ the matrices $A-R\left(\lambda_{i} E_{i i}\right) R^{-1}$ and $A-2 R\left(\lambda_{i} E_{i i}\right) R^{-1}$ are potents, and, therefore, $-\theta\left(R\left(\lambda_{i} E_{i i}\right) R^{-1}\right)$ and $-2 \theta\left(R\left(\lambda_{i} E_{i i}\right) R^{-1}\right)$ are potents as well; but, then $\theta\left(R\left(\lambda_{i} E_{i i}\right) R^{-1}\right)=0$. Thus, we proved that $\theta\left(R E_{i i} R^{-1}\right)=0$ for every $i \in\{1,2, \ldots, k\}$.

Now pick $j \in\{k+1, \ldots, n\}$. The matrices $R\left(E_{11}+E_{1 j}\right) R^{-1}$ and $R\left(E_{11}+E_{j 1}\right) R^{-1}$ are projections, so it follows that $\theta\left(R\left(E_{11}+E_{1 j}\right) R^{-1}\right)=$ $\theta\left(R E_{1 j} R^{-1}\right)$ and $\theta\left(R E_{j 1} R^{-1}\right)$ are potents; however, as $R E_{1 j} R^{-1}$ and $R E_{j 1} R^{-1}$ are nilpotents, $\theta\left(R E_{1 j} R^{-1}\right)$ and $\theta\left(R E_{j 1} R^{-1}\right)$ are nilpotents too (Step 1). Thus, $\theta\left(R E_{1 j} R^{-1}\right)=\theta\left(R E_{j 1} R^{-1}\right)=0$. Since $R\left(E_{11}-E_{1 j}+E_{j 1}-E_{j j}\right) R^{-1}$ is nilpotent, we have that

$$
\theta\left(R\left(E_{11}-E_{1 j}+E_{j 1}-E_{j j}\right) R^{-1}\right)=-\theta\left(R E_{j j} R^{-1}\right)
$$

is nilpotent; however, $\theta\left(R E_{j j} R^{-1}\right)$ is also a potent since $R E_{j j} R^{-1}$ is a potent; hence, $\theta\left(R E_{j j} R^{-1}\right)=0$. Thus we proved that $\theta\left(R E_{i i} R^{-1}\right)=0$ for every $i \in\{1,2, \ldots, n\}$, which clearly contradicts the assertion of Step 3 .

Step 5. If $N$ is a nilpotent and $N \neq 0$, then $\theta(N) \neq 0$.

Proof of Step 5. It can be easily shown that there exists a nilpotent matrix $M$ such that $M+N$ is a nonzero potent (for instance, if $N=E_{12}+E_{23}+\cdots+$ $E_{k-1, k}$, then these conditions are satisfied by $M=E_{k 1}$; a general case then 
follows by using a Jordan form of $N)$. Thus $\theta(M+N)$ is a nonzero potent by Step 4; hence, $\theta(N)$ cannot be zero, for otherwise it would follow that $\theta(M+N)=\theta(M)$ is a nilpotent (Step 1).

Step 6. Let $\Omega$ be the algebra of upper triangular matrices. There exists an invertible matrix $T$ such that $\theta(\Omega) \subseteq T \Omega T^{-1}$.

Proof of Step 6. Let $\Omega_{0}$ be the algebra of strictly upper triangular matrices. $\Omega_{0}$ is the space of nilpotents and its dimension is $n(n-1) / 2$; by Steps 1 and 5 , the same is true for $\theta\left(\Omega_{0}\right)$. Therefore, by a result of Gerstenhaber [5] there exists an invertible $T \in M_{n}$ such that $\theta\left(\Omega_{0}\right)=T \Omega_{0} T^{-1}$. The assertion will be proved by showing that $\theta$ maps diagonal matrices into the space $T \Omega T^{-1}$; thus, we must show that $T^{-1} \theta(A) T$ is an upper triangular matrix for any diagonal matrix $A$. It suffices to consider the case when $A$ is potent and its entries are mutually different (namely, the set of such matrices spans the space of diagonal matrices). For any $N \in \Omega_{0}$ we have $A+N \in \pi$. Hence $\theta(A)+\theta(N) \in$ $\pi$, so $T^{-1} \theta(A) T+T^{-1} \theta(N) T \in \pi$. Since $N$ is an arbitrary matrix in $\Omega_{0}$ and $\theta\left(\Omega_{0}\right)=T \Omega_{0} T^{-1}$, it follows that $T^{-1} \theta(A) T+\Omega_{0} \subseteq \pi$. Using standard arguments one shows that this implies that the matrix $T^{-1} \theta(A) T$ is upper triangular.

Step 7. $\theta(I)=c I$, and $c$ is a root of unity.

Proof of Step 7. Define $\sigma: M_{n} \rightarrow M_{n}$ by $\sigma(A)=T^{-1} \theta(A) T$. Obviously, $\sigma$ preserves potents, and, by the previous step, $\sigma(\Omega) \subseteq \Omega$.

Pick integers $i, j, 1 \leq i, j \leq n, i \neq j$. Since $E_{i i}, E_{j j}, E_{i i}+E_{j j}$, and $E_{i i}-E_{j j}$ are potent and upper triangular matrices, the same is true for the matrices $A_{i}=\sigma\left(E_{i i}\right), A_{j}=\sigma\left(E_{j j}\right), A_{i}+A_{j}$, and $A_{i}-A_{j}$. Thus, their diagonal entries are either roots of unity or zero. This clearly yields that $A_{i}$ and $A_{j}$ cannot simultaneously have nonzero entries in positions $(k, k), 1 \leq k \leq n$. However, since the $A_{i}$ 's are nonzero potents, each of them has at least one nonzero entry on a diagonal; thus, it has exactly one. By Step 2 it follows that this entry equals $c$ for any $i$. Thus, we proved that for any $i \in\{1,2, \ldots, n\}$ there exists an integer $k(i) \in\{1,2, \ldots, n\}$ and a matrix $N_{i} \in \Omega_{0}$ such that $\sigma\left(E_{i i}\right)=c E_{k(i), k(i)}+N_{i}$. Note also that $k(i) \neq k(j)$ if $i \neq j$. Hence,

$$
\sigma(I)=\sigma\left(\sum_{i=1}^{n} E_{i i}\right)=\sum_{i=1}^{n}\left(c E_{k(i), k(i)}+N_{i}\right)=c I+N,
$$

where $N \in \Omega_{0}$. Since $\sigma(I)$ is potent, it follows easily that $N=0$ and $c$ is a root of unity. Finally, note that $\sigma(I)=c I$ implies $\theta(I)=c I$.

Define a mapping $\psi: M_{n} \rightarrow M_{n}$ by $\psi(A)=c^{-1} \theta(A)$. As we have proved that (ii) implies (iii), the proof of the theorem will be complete by showing that $\psi\left(\pi_{2}\right) \subseteq \pi_{2}$. Thus, our last step is

Step 8. $\psi$ preserves projections.

Proof of Step 8. By Step 7, $\psi(I)=I$ and $\psi$ preserves potents. Pick a projection $P$, and let us show that $\psi(P)$ is a projection. Since $\psi(P)$ is a potent, it suffices to show that an arbitrary eigenvalue $\lambda$ of $\psi(P)$ is either 1 or 0 . Since $P, I-P$, and $I-2 P$ are potents, $\psi(P), I-\psi(P)$, and $I-2 \psi(P)$ are potents too, so 
it follows that each of the numbers $\lambda, 1-\lambda$, and $1-2 \lambda$ is either a root of unity or zero. The only two possibilities are that $\lambda$ is either 1 or 0 . The proof is thus complete.

\section{REFERENCES}

1. P. Botta, S. Pierce, and W. Watkins, Linear transformations that preserve the nilpotent matrices, Pacific J. Math. 104 (1983), 39-46.

2. M. Brešar and P. Šemrl, Mappings which preserve idempotents, local automorphisms, and local derivations, Canad. J. Math. (to appear).

3. J. Dieudonné, Sur une généralisation du groupe orthogonal à quatre variables, Arch. Math. 1 (1948), 282-287.

4. J. Dixon, Rigid embeddings of simple groups in the general linear group, Canad. J. Math. 29 (1977), 384-391.

5. M. Gerstenhaber, On nilalgebras and linear varieties of nilpotent matrices I, Amer. J. Math. 80 (1958), 614-622.

6. I. N. Herstein, Topics in ring theory, Univ. of Chicago Press, Chicago, 1969.

7. H. G. Jacob, Coherence invariant mappings on Kronecker products, Amer. J. Math. 77 (1955), 177-189.

8. D. R. Larson and A. R. Sourour, Local derivations and local automorphisms of $B(X)$, Proc. Sympos. Pure Math., vol. 51, part 2, Amer. Math. Soc., Providence, RI, 1990, pp. 187-194.

9. M. Marcus and B. Moyls, Transformations on tensor product spaces, Pacific J. Math. 9 (1959), 1215-1221.

Department of Mathematics, University of Maribor, PF, KorošKa 160, 62000 Maribor, SLOVENIA

Department of Mathematics, University of Ljubljana, Jadranska 19, 61000 Ljubljana, SLOVENIA 